Supporting Information

\title{
Lead Isotopes as A New Tracer for Detecting Coal Fly Ash in the Environment
}

Zhen Wang^, Gary S. Dwyer ${ }^{\wedge}$, Drew S. Coleman\#, Avner Vengosh ${ }^{\wedge *}$

${ }^{\wedge}$ Division of Earth and Ocean Sciences, Nicholas School of the Environment, Duke University, Durham, NC 27708

\#Department of Geological Sciences, University of North Carolina, Chapel Hill, NC 27599

*Corresponding author

(vengosh@duke.edu)

Contents

1. Analytical Techniques

2. Tables (Table S1 to Table S5)

3. Figures (Figure $\mathrm{S} 1$ to Figure S3)

4. References
Page

S2

S3

S14

S17 


\section{Analytical Techniques}

\section{Sample digestion procedures:}

1. Weigh $10 \pm 0.5 \mathrm{mg}$ sample in a $5 \mathrm{~mL}$ Teflon beaker;

2. Add $0.1 \mathrm{~mL} 29 \mathrm{M} \mathrm{HF}$ and $2 \mathrm{~mL} 15 \mathrm{M} \mathrm{HNO}_{3}$ (optima grade);

3. Cap and shake the beaker gently, and place it on the hotplate at $160{ }^{\circ} \mathrm{C}$ for at least 24 hours;

4. Turn off hotplate and let the beaker cool down;

5. Slowly uncap the beaker and place both the cap and beaker on the hotplate at $140{ }^{\circ} \mathrm{C}$ for dry down;

6. Add $2 \mathrm{~mL} 6 \mathrm{M} \mathrm{HCl}$ to dissolve the dry-down residue, cap the beaker tightly and place it on the hotplate at $160^{\circ} \mathrm{C}$ for at least 24 hours.

\section{Lead column separation procedures:}

1. Take aliquots from digested solutions and dry down at $110^{\circ} \mathrm{C}$;

2. Redissolve in $\sim 500 \mu \mathrm{L} 1.1 \mathrm{~N} \mathrm{HBr}$;

3. Rinse resin with DI water, $500 \mu \mathrm{L} 6 \mathrm{~N} \mathrm{HCl}$, and precondition resin in $250 \mu 11.1 \mathrm{~N} \mathrm{HBr}$;

4. Load sample solutions into resin;

5. Rinse sample with $\sim 500 \mu \mathrm{L} \mathrm{HBr}$ and $\sim 250 \mu \mathrm{L} 2 \mathrm{~N} \mathrm{HCl}$;

6. Elute $\mathrm{Pb}$ using $\sim 500 \mu \mathrm{l} 6 \mathrm{~N} \mathrm{HCl}$;

7. Dry down elution, redissolve in $\mathrm{HBr}$ and repeat from step 3;

8. Add one drop of $\mathrm{H}_{3} \mathrm{PO}_{4}$ and then dry down completely. 
Table S1: Sample collection information of fly ash samples analyzed in this study. ESP: electrostatic precipitator; mech: mechanical; baghouse: baghouse filter.

\begin{tabular}{|c|c|c|c|c|}
\hline Sample ID & Sampled Power Plant & Sample Type & $\begin{array}{c}\text { Sampling } \\
\text { Year }\end{array}$ & Coal Source \\
\hline \multicolumn{5}{|c|}{ Appalachian Basin, Pennsylvanian age, Bituminous coal } \\
\hline APP-1 & E.W. Brown, KY & ESP fly ash & 2004 & \multirow{3}{*}{$\begin{array}{c}\text { Central } \\
\text { Appalachian }\end{array}$} \\
\hline APP-2 & E.W. Brown, KY & ESP fly ash & 2004 & \\
\hline APP-3 & E.W. Brown, KY & ESP fly ash & 2004 & \\
\hline APP-4 & Cooper, KY & ESP fly ash & 2007 & \multirow{9}{*}{$\begin{array}{c}\text { Eastern } \\
\text { Kentucky \& } \\
\text { Central West } \\
\text { Virginia }\end{array}$} \\
\hline APP-5 & Cooper, KY & ESP fly ash & 2007 & \\
\hline APP-6 & Big Sandy, KY & ESP fly ash & 2007 & \\
\hline APP-7 & Big Sandy, KY & ESP fly ash & 2007 & \\
\hline APP-8 & Big Sandy, KY & ESP fly ash & 2007 & \\
\hline APP-9 & Big Sandy, KY & ESP fly ash & 2007 & \\
\hline APP-10 & Big Sandy, KY & ESP fly ash & 2007 & \\
\hline APP-11 & Big Sandy, KY & ESP fly ash & 2007 & \\
\hline APP-12 & Big Sandy, KY & ESP fly ash & 2007 & \\
\hline APP-13 & TVA Bull Run, TN & fly ash & 2011 & \multirow{2}{*}{$\begin{array}{c}\text { Central } \\
\text { Appalachian }\end{array}$} \\
\hline APP-14 & John Sevier, TN & fly ash & 2011 & \\
\hline APP-15 & Cooper, KY & mech fly ash & 2013 & \multirow{2}{*}{$\begin{array}{c}\text { Southeastern } \\
\text { Kentucky }\end{array}$} \\
\hline APP-16 & Cooper, KY & ESP fly ash & 2013 & \\
\hline \multicolumn{5}{|c|}{ Illinois Basin, Pennsylvanian age, Bituminous coal } \\
\hline ILL-1 & Trimble, KY & ESP fly ash & 2012 & \multirow{10}{*}{$\begin{array}{l}\text { Western } \\
\text { Kentucky }\end{array}$} \\
\hline ILL-2 & Trimble, KY & ESP fly ash & 2012 & \\
\hline ILL-3 & Trimble, KY & ESP fly ash & 2012 & \\
\hline ILL-4 & Trimble, KY & ESP fly ash & 2012 & \\
\hline ILL-5 & Green River, KY & ESP fly ash & 2012 & \\
\hline ILL-6 & Green River, KY & ESP fly ash & 2012 & \\
\hline ILL-7 & D.B. Wilson, KY & ESP fly ash & 2012 & \\
\hline ILL-8 & D.B. Wilson, KY & ESP fly ash & 2012 & \\
\hline ILL-9 & Coleman, KY & ESP fly ash & 2012 & \\
\hline ILL-10 & Coleman, KY & ESP fly ash & 2012 & \\
\hline ILL-11 & E.W. Brown, KY & ESP fly ash & 2012 & \multirow{4}{*}{ Indiana } \\
\hline ILL-12 & E.W. Brown, KY & ESP fly ash & 2012 & \\
\hline ILL-13 & E.W. Brown, KY & ESP fly ash & 2012 & \\
\hline ILL-14 & E.W. Brown, KY & ESP fly ash & 2012 & \\
\hline ILL-15 & Trimble, KY & ESP fly ash & 2013 & \multirow{5}{*}{$\begin{array}{l}\text { Western } \\
\text { Kentucky }\end{array}$} \\
\hline ILL-16 & Trimble, KY & ESP fly ash & 2013 & \\
\hline ILL-17 & Trimble, KY & ESP fly ash & 2013 & \\
\hline ILL-18 & Trimble, KY & ESP fly ash & 2013 & \\
\hline ILL-19 & Trimble, KY & ESP fly ash & 2013 & \\
\hline ILL-20 & E.W. Brown, KY & ESP fly ash & 2013 & Indiana \\
\hline
\end{tabular}




\begin{tabular}{|c|c|c|c|c|}
\hline ILL-21 & E.W. Brown, KY & ESP fly ash & 2013 & \\
\hline ILL-22 & E.W. Brown, KY & ESP fly ash & 2013 & \\
\hline \multicolumn{5}{|c|}{ Powder River Basin, Paleocene and Late Cretaceous age, Subbituminous coal } \\
\hline PRB-1 & Kingston, TN & fly ash & 2011 & \multirow{4}{*}{$\begin{array}{l}\text { Powder } \\
\text { River }\end{array}$} \\
\hline PRB-2 & Kingston, TN & fly ash & 2011 & \\
\hline PRB-3 & Kingston, TN & fly ash & 2011 & \\
\hline PRB-4 & Kingston, TN & fly ash & 2011 & \\
\hline PRB-5 & Shawnee, KY & mech fly ash & 2012 & \multirow[b]{2}{*}{ Wyoming } \\
\hline PRB-6 & Shawnee, KY & $\begin{array}{c}\text { baghouse fly } \\
\text { ash }\end{array}$ & 2012 & \\
\hline PRB-7 & Meramec, MO & fly ash & 2013 & $\begin{array}{l}\text { Powder } \\
\text { River }\end{array}$ \\
\hline
\end{tabular}


Table S2: Raw Pb stable isotope ratios of NIST SRM 981 measured in this study. Mass bias was calculated by normalizing the raw data to the expected values. 2SD (standard deviation) represents the uncertainties.

\begin{tabular}{|c|c|c|c|c|c|}
\hline No. & ${ }^{208} \mathrm{~Pb} /{ }^{204} \mathrm{~Pb}$ & ${ }^{207} \mathrm{~Pb} /{ }^{204} \mathrm{~Pb}$ & ${ }^{206} \mathrm{~Pb} /{ }^{204} \mathrm{~Pb}$ & ${ }^{208} \mathrm{~Pb} /{ }^{206} \mathrm{~Pb}$ & ${ }^{206} \mathrm{~Pb} /{ }^{207} \mathrm{~Pb}$ \\
\hline 1 & 36.4848 & 15.4234 & 16.8857 & 2.1607 & 1.0948 \\
\hline 2 & 36.4904 & 15.4259 & 16.8866 & 2.1609 & 1.0948 \\
\hline 3 & 36.4853 & 15.4239 & 16.8862 & 2.1607 & 1.0948 \\
\hline 4 & 36.4901 & 15.4248 & 16.8872 & 2.1608 & 1.0948 \\
\hline 5 & 36.4850 & 15.4233 & 16.8856 & 2.1607 & 1.0948 \\
\hline 6 & 36.4759 & 15.4213 & 16.8834 & 2.1605 & 1.0949 \\
\hline 7 & 36.4984 & 15.4262 & 16.8888 & 2.1611 & 1.0947 \\
\hline 8 & 36.4916 & 15.4265 & 16.8873 & 2.1609 & 1.0947 \\
\hline 9 & 36.4926 & 15.4267 & 16.8875 & 2.1609 & 1.0947 \\
\hline 10 & 36.4893 & 15.4245 & 16.8869 & 2.1608 & 1.0948 \\
\hline 11 & 36.4865 & 15.4235 & 16.8858 & 2.1608 & 1.0948 \\
\hline 12 & 36.4872 & 15.4238 & 16.8861 & 2.1608 & 1.0948 \\
\hline 13 & 36.4952 & 15.4272 & 16.8880 & 2.1610 & 1.0947 \\
\hline 14 & 36.4859 & 15.4237 & 16.8860 & 2.1607 & 1.0948 \\
\hline 15 & 36.4864 & 15.4235 & 16.8858 & 2.1608 & 1.0948 \\
\hline 16 & 36.4930 & 15.4246 & 16.8870 & 2.1609 & 1.0948 \\
\hline 17 & 36.4930 & 15.4264 & 16.8871 & 2.1610 & 1.0947 \\
\hline 18 & 36.4846 & 15.4231 & 16.8854 & 2.1607 & 1.0948 \\
\hline 19 & 36.4859 & 15.4237 & 16.8860 & 2.1607 & 1.0948 \\
\hline 20 & 36.4792 & 15.4218 & 16.8839 & 2.1606 & 1.0948 \\
\hline 21 & 36.4802 & 15.4226 & 16.8848 & 2.1605 & 1.0948 \\
\hline Mean & 36.4876 & 15.4245 & 16.8862 & 2.1608 & 1.0948 \\
\hline 2SD & 0.0109 & 0.0032 & 0.0026 & 0.0003 & 0.0001 \\
\hline Expected values $^{1}$ & 36.7184 & 15.4957 & 16.9406 & 2.1675 & 1.0932 \\
\hline Mass bias (\% per amu) & 0.16 & 0.15 & 0.16 & 0.15 & 0.14 \\
\hline
\end{tabular}


Table $\mathrm{S} 3: \mathrm{Pb}$ stable isotope ratios, $\mathrm{Pb}$, Th, and $\mathrm{U}$ concentrations, and ${ }^{210} \mathrm{~Pb}$ activities and ${ }^{210} \mathrm{~Pb} /{ }^{226} \mathrm{Ra}$ ratios of fly ash samples derived from coals of three coal-producing basins from the U.S. SD: standard deviation.

\begin{tabular}{|c|c|c|c|c|c|c|c|c|c|c|}
\hline $\begin{array}{c}\text { Sample } \\
\text { ID }\end{array}$ & ${ }^{208} \mathrm{~Pb} /{ }^{204} \mathrm{~Pb}$ & ${ }^{207} \mathrm{~Pb} /{ }^{204} \mathrm{~Pb}$ & ${ }^{206} \mathrm{~Pb} /{ }^{204} \mathrm{~Pb}$ & ${ }^{208} \mathrm{~Pb} /{ }^{206} \mathrm{~Pb}$ & ${ }^{206} \mathrm{~Pb} /{ }^{207} \mathrm{~Pb}$ & $\begin{array}{c}\mathrm{Pb} \\
(\mathrm{mg} / \mathrm{kg})\end{array}$ & $\begin{array}{c}\text { Th } \\
(\mathrm{mg} / \mathrm{kg})\end{array}$ & $\begin{array}{c}\mathrm{U} \\
(\mathrm{mg} / \mathrm{kg})\end{array}$ & ${ }^{210} \mathrm{~Pb}(\mathrm{~Bq} / \mathbf{k g})^{2}$ & ${ }^{210} \mathrm{~Pb} /{ }^{226} \mathbf{R a}^{2}$ \\
\hline APP-1 & 39.0050 & 15.6785 & 19.2592 & 2.0253 & 1.2284 & 39.24 & 24.64 & 11.47 & 112 & 0.75 \\
\hline APP-2 & 38.8460 & 15.6816 & 19.2568 & 2.0172 & 1.2280 & 76.27 & 28.73 & 15.08 & 180 & 0.99 \\
\hline APP-3 & 38.8317 & 15.6798 & 19.2280 & 2.0195 & 1.2263 & 123.32 & 27.94 & 16.74 & 256 & 1.39 \\
\hline APP-4 & 38.8407 & 15.6868 & 19.1660 & 2.0266 & 1.2217 & 133.88 & 28.66 & 19.89 & & \\
\hline APP-5 & 38.8104 & 15.6807 & 19.0983 & 2.0321 & 1.2180 & 60.89 & 23.91 & 10.95 & & \\
\hline APP-6 & 38.8187 & 15.6791 & 19.0666 & 2.0360 & 1.2161 & 84.40 & 30.06 & 13.04 & 186 & 1.06 \\
\hline APP-7 & 38.8278 & 15.6781 & 19.0764 & 2.0354 & 1.2167 & 84.18 & 30.02 & 12.99 & 194 & 1.08 \\
\hline APP-8 & 38.8399 & 15.6845 & 19.0881 & 2.0348 & 1.2170 & 82.71 & 29.26 & 12.82 & 180 & 1.06 \\
\hline APP-9 & 38.7514 & 15.6607 & 18.9771 & 2.0420 & 1.2118 & 83.73 & 31.05 & 11.88 & 162 & 1.05 \\
\hline APP-10 & 38.7454 & 15.6639 & 18.9647 & 2.0430 & 1.2107 & 72.69 & 28.18 & 10.32 & 142 & 0.99 \\
\hline APP-11 & 38.7672 & 15.6737 & 18.9747 & 2.0431 & 1.2107 & 114.32 & 28.55 & 14.32 & 189 & 1.16 \\
\hline APP-12 & 38.8245 & 15.6862 & 19.0073 & 2.0426 & 1.2117 & 118.06 & 29.37 & 14.93 & 205 & 1.31 \\
\hline APP-13 & 38.7853 & 15.6664 & 19.0128 & 2.0400 & 1.2137 & 80.42 & 29.29 & 12.05 & 310 & 1.33 \\
\hline APP-14 & 38.8010 & 15.6735 & 19.0496 & 2.0368 & 1.2154 & 77.08 & 24.95 & 10.75 & 142 & 1.06 \\
\hline APP-15 & 38.7546 & 15.6618 & 18.9786 & 2.0420 & 1.2118 & 47.08 & 20.04 & 10.14 & 121 & 0.77 \\
\hline APP-16 & 38.7940 & 15.6801 & 19.1014 & 2.0310 & 1.2183 & 136.30 & 26.05 & 18.43 & 324 & 1.64 \\
\hline Mean & 38.8152 & 15.6759 & 19.0816 & 2.0342 & 1.2173 & 88.41 & 27.54 & 13.49 & 193 & 1.12 \\
\hline Median & 38.8145 & 15.6788 & 19.0715 & 2.0357 & 1.2164 & 83.22 & 28.60 & 12.91 & 183 & 1.06 \\
\hline SD & 0.0607 & 0.0085 & 0.0999 & 0.0084 & 0.0060 & 29.08 & 2.89 & 2.90 & 64 & 0.24 \\
\hline ILL-1 & 38.6719 & 15.6799 & 19.2421 & 2.0097 & 1.2272 & 93.84 & 19.32 & 19.86 & 259 & 1.16 \\
\hline ILL-2 & 38.6829 & 15.6814 & 19.2205 & 2.0126 & 1.2257 & 101.75 & 19.50 & 18.71 & 265 & 1.28 \\
\hline ILL-3 & 38.6723 & 15.6733 & 19.1730 & 2.0170 & 1.2233 & 98.53 & 19.10 & 17.03 & 246 & 1.30 \\
\hline ILL-4 & 38.6299 & 15.6800 & 19.3159 & 1.9999 & 1.2319 & 105.73 & 18.40 & 24.09 & 318 & 1.26 \\
\hline ILL-5 & 38.6565 & 15.6692 & 18.9740 & 2.0374 & 1.2109 & & & & & \\
\hline ILL-6 & 38.6556 & 15.6694 & 18.9930 & 2.0353 & 1.2120 & & & & & \\
\hline ILL-7 & 38.7829 & 15.7155 & 19.6028 & 1.9784 & 1.2473 & & & & & \\
\hline
\end{tabular}




\begin{tabular}{|c|c|c|c|c|c|c|c|c|c|c|}
\hline $\begin{array}{c}\text { Sample } \\
\text { ID }\end{array}$ & ${ }^{208} \mathrm{~Pb} /{ }^{204} \mathrm{~Pb}$ & ${ }^{207} \mathrm{~Pb} /{ }^{204} \mathrm{~Pb}$ & ${ }^{206} \mathrm{~Pb} /{ }^{204} \mathrm{~Pb}$ & ${ }^{208} \mathrm{~Pb} /{ }^{206} \mathrm{~Pb}$ & ${ }^{206} \mathrm{~Pb} /{ }^{207} \mathrm{~Pb}$ & $\begin{array}{c}\mathrm{Pb} \\
(\mathrm{mg} / \mathrm{kg})\end{array}$ & $\begin{array}{c}\text { Th } \\
(\mathrm{mg} / \mathrm{kg})\end{array}$ & $\begin{array}{c}\mathrm{U} \\
(\mathrm{mg} / \mathrm{kg})\end{array}$ & ${ }^{210} \mathrm{~Pb}(\mathrm{~Bq} / \mathrm{kg})^{2}$ & ${ }^{210} \mathbf{P b} /{ }^{226} \mathbf{R a}^{2}$ \\
\hline ILL-8 & 38.7777 & 15.7141 & 19.6090 & 1.9776 & 1.2479 & & & & & \\
\hline ILL-9 & 38.6744 & 15.7140 & 19.5430 & 1.9789 & 1.2437 & & & & & \\
\hline ILL-10 & 38.6625 & 15.7126 & 19.5663 & 1.9760 & 1.2453 & & & & & \\
\hline ILL-11 & 38.7277 & 15.6767 & 19.0739 & 2.0304 & 1.2168 & 102.82 & 20.21 & 13.19 & 164 & 1.13 \\
\hline ILL-12 & 38.7117 & 15.6746 & 19.0635 & 2.0307 & 1.2162 & 140.77 & 19.53 & 15.02 & 206 & 1.41 \\
\hline ILL-13 & 38.6996 & 15.6735 & 19.0356 & 2.0330 & 1.2144 & 213.69 & 20.71 & 17.72 & 299 & 1.77 \\
\hline ILL-14 & 38.6433 & 15.6614 & 19.0006 & 2.0339 & 1.2132 & 308.00 & 22.74 & 22.20 & 420 & 2.13 \\
\hline ILL-15 & 38.6064 & 15.6897 & 19.4218 & 1.9878 & 1.2379 & 103.32 & 16.31 & 24.43 & 384 & 1.34 \\
\hline ILL-16 & 38.5971 & 15.6878 & 19.4179 & 1.9878 & 1.2379 & 113.86 & 16.29 & 25.58 & 386 & 1.39 \\
\hline ILL-17 & 38.6154 & 15.6925 & 19.4237 & 1.9881 & 1.2377 & 118.08 & 16.35 & 26.62 & 398 & 1.48 \\
\hline ILL-18 & 38.5942 & 15.6856 & 19.4152 & 1.9879 & 1.2377 & 128.96 & 16.17 & 26.79 & 453 & 1.61 \\
\hline ILL-19 & 38.6074 & 15.6888 & 19.4145 & 1.9886 & 1.2374 & 129.21 & 16.22 & 26.08 & 488 & 1.77 \\
\hline ILL-20 & 38.6244 & 15.6651 & 18.9675 & 2.0364 & 1.2109 & 137.14 & 19.16 & 19.35 & 187 & 0.86 \\
\hline ILL-21 & 38.6130 & 15.6616 & 18.9601 & 2.0365 & 1.2106 & 200.58 & 20.71 & 21.70 & 283 & 1.22 \\
\hline ILL-22 & 38.6368 & 15.6657 & 18.9236 & 2.0417 & 1.2079 & 114.29 & 15.92 & 13.88 & 130 & 0.91 \\
\hline Mean & 38.6611 & 15.6833 & 19.2435 & 2.0093 & 1.2270 & 138.16 & 18.54 & 20.77 & 305 & 1.38 \\
\hline Median & 38.6560 & 15.6800 & 19.2313 & 2.0112 & 1.2265 & 116.19 & 19.13 & 20.78 & 291 & 1.32 \\
\hline SD & 0.0534 & 0.0173 & 0.2369 & 0.0244 & 0.0139 & 56.90 & 2.09 & 4.60 & 107 & 0.33 \\
\hline PRB-1 & 38.9738 & 15.6940 & 19.1529 & 2.0349 & 1.2205 & 59.12 & 27.47 & 10.35 & 123 & 1.00 \\
\hline PRB-2 & 38.9830 & 15.6945 & 19.1865 & 2.0318 & 1.2224 & 56.23 & 27.83 & 10.72 & 123 & 0.98 \\
\hline PRB-3 & 38.9892 & 15.6949 & 19.2152 & 2.0291 & 1.2242 & 50.09 & 27.21 & 10.03 & 108 & 0.87 \\
\hline PRB-4 & 39.0978 & 15.7161 & 19.2506 & 2.0310 & 1.2248 & 26.50 & 22.95 & 8.34 & 72 & 0.71 \\
\hline PRB-5 & 39.1706 & 15.6915 & 19.2517 & 2.0347 & 1.2269 & 25.26 & 25.50 & 9.12 & 70 & 0.64 \\
\hline PRB-6 & 39.0669 & 15.6791 & 19.1974 & 2.0350 & 1.2244 & 74.97 & 24.75 & 11.91 & 179 & 1.32 \\
\hline PRB-7 & 39.3406 & 15.7307 & 19.6022 & 2.0069 & 1.2461 & 38.86 & 22.20 & 7.77 & 98 & 0.94 \\
\hline Mean & 39.0888 & 15.7001 & 19.2652 & 2.0290 & 1.2271 & 47.29 & 25.41 & 9.75 & 110 & 0.92 \\
\hline Median & 39.0669 & 15.6945 & 19.2152 & 2.0318 & 1.2244 & 50.09 & 25.50 & 10.03 & 108 & 0.94 \\
\hline SD & 0.1323 & 0.0173 & 0.1527 & 0.0100 & 0.0086 & 18.18 & 2.24 & 1.44 & 37 & 0.22 \\
\hline
\end{tabular}


Table S4: Previously reported $\mathrm{Pb}$ stable isotopes data of other anthropogenic and geogenic $\mathrm{Pb}$ sources in the U.S. SD: standard deviation.

\begin{tabular}{|c|c|c|c|}
\hline Sample & ${ }^{206} \mathrm{~Pb} /{ }^{204} \mathrm{~Pb}$ & ${ }^{208} \mathrm{~Pb} /{ }^{206} \mathrm{~Pb}$ & ${ }^{206} \mathrm{~Pb} /{ }^{207} \mathrm{~Pb}$ \\
\hline \multicolumn{4}{|c|}{ Lead ores ${ }^{3,4}$} \\
\hline Balmat & & 2.1623 & 1.0878 \\
\hline Shafter & & 2.1074 & 1.1522 \\
\hline Austinville/Ivanhoe & & 2.0370 & 1.2127 \\
\hline Central TN & & 2.0052 & 1.2452 \\
\hline Eastern TN & & 2.0113 & 1.2402 \\
\hline Southeast MO & & 1.8885 & 1.3390 \\
\hline Tri-State & & 1.8788 & 1.3754 \\
\hline $\begin{array}{c}\text { Upper Mississippi } \\
\text { Valley }\end{array}$ & & 1.8698 & 1.4060 \\
\hline Mean & & 1.9950 & 1.2573 \\
\hline SD & & 0.1091 & 0.1102 \\
\hline \multicolumn{4}{|c|}{ Leaded gasoline $^{5-7}$} \\
\hline & 18.583 & 2.0546 & 1.1895 \\
\hline & 18.604 & 2.0558 & 1.1912 \\
\hline & 18.506 & 2.0664 & 1.1862 \\
\hline & 18.594 & 2.0593 & 1.1903 \\
\hline & 18.658 & 2.0655 & 1.1959 \\
\hline & 18.570 & 2.0558 & 1.1902 \\
\hline & 18.631 & 2.0505 & 1.1933 \\
\hline & 18.700 & 2.0482 & 1.1965 \\
\hline & 18.657 & 2.0507 & 1.1948 \\
\hline & 18.580 & 2.0601 & 1.1889 \\
\hline & 18.526 & 2.0633 & 1.1877 \\
\hline & 18.550 & 2.0635 & 1.1876 \\
\hline & 18.497 & 2.0681 & 1.1854 \\
\hline & & 2.1500 & 1.1170 \\
\hline & & 2.0400 & 1.1990 \\
\hline & & 2.0480 & 1.1930 \\
\hline & & 2.0600 & 1.1900 \\
\hline & & 2.0820 & 1.1710 \\
\hline & & 2.1090 & 1.1520 \\
\hline & & 2.0980 & 1.1600 \\
\hline & & 2.1180 & 1.1420 \\
\hline & & 2.1080 & 1.1570 \\
\hline & & 2.1100 & 1.1450 \\
\hline Mean & 18.5889 & 2.0733 & 1.1784 \\
\hline SD & 0.0613 & 0.0283 & 0.0220 \\
\hline
\end{tabular}




\begin{tabular}{|c|c|c|c|}
\hline Sample & ${ }^{206} \mathrm{~Pb} /{ }^{204} \mathrm{~Pb}$ & ${ }^{208} \mathrm{~Pb} /{ }^{206} \mathrm{~Pb}$ & ${ }^{206} \mathrm{~Pb} /{ }^{207} \mathrm{~Pb}$ \\
\hline \multicolumn{4}{|c|}{ Lead-based paint $^{8-10}$} \\
\hline & 18.2482 & 2.1260 & 1.1334 \\
\hline & 18.6220 & 2.1030 & 1.1489 \\
\hline & 19.1205 & 2.0340 & 1.2332 \\
\hline & 18.1159 & 2.1050 & 1.1602 \\
\hline & 18.5185 & 2.0970 & 1.1650 \\
\hline & 18.4502 & 2.0750 & 1.1752 \\
\hline & 17.8571 & 2.1050 & 1.1486 \\
\hline & 18.7970 & 2.0950 & 1.1703 \\
\hline & 18.9036 & 2.0320 & 1.2061 \\
\hline & 18.7617 & 2.0740 & 1.1899 \\
\hline & 18.2815 & 2.1230 & 1.1375 \\
\hline & 16.93 & & 1.0940 \\
\hline & 17.57 & & 1.1290 \\
\hline & 17.45 & & 1.1290 \\
\hline & 17.70 & & 1.1400 \\
\hline & 18.88 & & 1.1900 \\
\hline & 18.72 & & 1.1970 \\
\hline & 18.79 & & 1.2050 \\
\hline & 18.04 & & 1.1510 \\
\hline & 18.17 & & 1.1590 \\
\hline & 17.77 & & 1.1330 \\
\hline & 18.32 & & 1.1630 \\
\hline & 18.19 & & 1.1659 \\
\hline & 18.44 & & 1.1822 \\
\hline & 18.25 & & 1.1710 \\
\hline & 18.50 & & 1.1855 \\
\hline & 18.49 & & 1.1849 \\
\hline & 18.14 & & 1.1655 \\
\hline & 17.71 & & 1.1406 \\
\hline Mean & 18.2667 & 2.0881 & 1.1639 \\
\hline SD & 0.499 & 0.0317 & 0.0292 \\
\hline \multicolumn{4}{|c|}{ Aerosols $^{11,12}$} \\
\hline \multirow{8}{*}{ East U.S. } & 18.88 & 2.0198 & 1.2140 \\
\hline & 19.02 & 2.0325 & 1.2105 \\
\hline & 19.01 & 2.0360 & 1.2080 \\
\hline & 19.39 & 2.0061 & 1.2314 \\
\hline & 19.06 & 2.0252 & 1.2159 \\
\hline & 19.05 & 2.0296 & 1.2131 \\
\hline & 18.69 & 2.0503 & 1.1955 \\
\hline & 18.33 & 2.0807 & 1.1733 \\
\hline
\end{tabular}




\begin{tabular}{|c|c|c|c|}
\hline & ${ }^{206} \mathrm{~Pb} /{ }^{204} \mathrm{~Pb}$ & ${ }^{208} \mathrm{~Pb} / 206 \mathrm{~Pb}$ & ${ }^{206} \mathrm{~Pb} /{ }^{207} \mathrm{~Pb}$ \\
\hline & 18.43 & 2.0664 & 1.1801 \\
\hline & 18.36 & 2.0724 & 1.1762 \\
\hline & 18.43 & 2.0687 & 1.1797 \\
\hline & 19.19 & 2.0141 & 1.2232 \\
\hline & 18.89 & 2.0542 & 1.1970 \\
\hline & 19.04 & 2.0279 & 1.2141 \\
\hline \multirow{5}{*}{ Mid U.S. } & 18.96 & 2.0289 & 1.2110 \\
\hline & 19.18 & 2.0123 & 1.2230 \\
\hline & 19.12 & 2.0156 & 1.2200 \\
\hline & 18.62 & 2.0502 & 1.1950 \\
\hline & 18.78 & 2.0341 & 1.2020 \\
\hline \multirow{20}{*}{ West U.S. } & 18.57 & 2.0589 & 1.1880 \\
\hline & 18.45 & 2.0655 & 1.1809 \\
\hline & 18.37 & 2.0740 & 1.1741 \\
\hline & 18.39 & 2.0772 & 1.1742 \\
\hline & 18.10 & 2.0936 & 1.1588 \\
\hline & 18.32 & 2.0919 & 1.1660 \\
\hline & 18.20 & 2.0994 & 1.1604 \\
\hline & 18.33 & 2.0782 & 1.1736 \\
\hline & 18.35 & 2.0773 & 1.1716 \\
\hline & 18.17 & 2.0841 & 1.1658 \\
\hline & 18.54 & 2.0582 & 1.1863 \\
\hline & 18.35 & 2.0733 & 1.1750 \\
\hline & 18.47 & 2.0680 & 1.1811 \\
\hline & 18.24 & 2.0817 & 1.1692 \\
\hline & 18.33 & 2.0717 & 1.1746 \\
\hline & 18.50 & 2.0721 & 1.1846 \\
\hline & 18.21 & 2.0979 & 1.1599 \\
\hline & 18.21 & 2.0860 & 1.1652 \\
\hline & 18.44 & 2.0741 & 1.1751 \\
\hline & 18.12 & 2.0912 & 1.1623 \\
\hline Mean & 18.59 & 2.0590 & 1.1879 \\
\hline SD & 0.36 & 0.0270 & 0.0211 \\
\hline \multicolumn{4}{|c|}{ Coals $^{13,14}$} \\
\hline & & 2.0640 & 1.1987 \\
\hline & & 2.0829 & 1.1907 \\
\hline & & 2.0625 & 1.1945 \\
\hline & & 2.0494 & 1.2109 \\
\hline & & 2.0694 & 1.1938 \\
\hline & & 2.0668 & 1.1960 \\
\hline & & 2.0447 & 1.2047 \\
\hline
\end{tabular}




\begin{tabular}{|c|c|c|c|}
\hline & ${ }^{206} \mathrm{~Pb} /{ }^{204} \mathrm{~Pb}$ & ${ }^{208} \mathrm{~Pb} / 206 \mathrm{~Pb}$ & ${ }^{206} \mathrm{~Pb} / 207 \mathrm{~Pb}$ \\
\hline & & 2.0464 & 1.2107 \\
\hline & & 2.0201 & 1.2215 \\
\hline & & 2.0526 & 1.2029 \\
\hline & 18.64 & 2.0730 & 1.1890 \\
\hline & 18.82 & 2.0619 & 1.1970 \\
\hline & 18.82 & 2.0623 & 1.2000 \\
\hline & 18.79 & 2.0627 & 1.1990 \\
\hline & 18.96 & 2.0442 & 1.2120 \\
\hline & 19.04 & 2.0284 & 1.2120 \\
\hline & 19.08 & 2.0446 & 1.2090 \\
\hline & 19.11 & 2.0190 & 1.2220 \\
\hline & 18.64 & 2.0799 & 1.1830 \\
\hline & 19.72 & 1.9643 & 1.2520 \\
\hline & 19.40 & 2.0346 & 1.2090 \\
\hline & 18.55 & 2.0855 & 1.1820 \\
\hline & 18.52 & 2.0777 & 1.1830 \\
\hline & 19.05 & 2.0375 & 1.2140 \\
\hline & 18.77 & 2.0683 & 1.1960 \\
\hline & 18.80 & 2.0636 & 1.1970 \\
\hline & 18.78 & 2.0803 & 1.1900 \\
\hline & 19.12 & 2.0375 & 1.2220 \\
\hline Mean & 18.92 & 2.0530 & 1.2033 \\
\hline SD & 0.30 & 0.0255 & 0.0150 \\
\hline & bsurface soi & & \\
\hline & & 2.0512 & 1.1933 \\
\hline & & 2.0548 & 1.1956 \\
\hline Virginia & & 2.0657 & 1.2090 \\
\hline & & 2.0542 & 1.2153 \\
\hline & & 2.0688 & 1.1737 \\
\hline & & 2.0462 & 1.2182 \\
\hline & & 2.0521 & 1.2228 \\
\hline West Viroinia & & 2.0576 & 1.2012 \\
\hline west vilginla & & 2.0443 & 1.2223 \\
\hline & & 2.0455 & 1.2210 \\
\hline & & 2.0480 & 1.2241 \\
\hline & & 2.0504 & 1.2230 \\
\hline & & 2.0624 & 1.2195 \\
\hline Kenturck & & 2.0482 & 1.2081 \\
\hline Rentucку & & 2.0354 & 1.2273 \\
\hline & & 2.0484 & 1.2195 \\
\hline & & 2.0438 & 1.2228 \\
\hline
\end{tabular}




\begin{tabular}{|c|c|c|c|}
\hline & ${ }^{206} \mathrm{~Pb} /{ }^{204} \mathrm{~Pb}$ & ${ }^{208} \mathrm{~Pb} / 206 \mathrm{~Pb}$ & ${ }^{206} \mathrm{~Pb} / 207 \mathrm{~Pb}$ \\
\hline & & 2.0353 & 1.2227 \\
\hline & & 2.0427 & 1.2164 \\
\hline & & 2.0350 & 1.2230 \\
\hline & & 2.0318 & 1.2210 \\
\hline & & 2.0184 & 1.2201 \\
\hline & & 2.0290 & 1.2277 \\
\hline & & 2.0317 & 1.2303 \\
\hline & & 2.0294 & 1.2117 \\
\hline & & 2.0397 & 1.2287 \\
\hline \multirow{7}{*}{ Illinois } & & 2.0414 & 1.2284 \\
\hline & & 2.0182 & 1.2567 \\
\hline & & 2.0068 & 1.2661 \\
\hline & & 2.0262 & 1.2432 \\
\hline & & 2.0322 & 1.2404 \\
\hline & & 2.0262 & 1.2406 \\
\hline & & 2.0239 & 1.2372 \\
\hline \multirow{4}{*}{ Indiana } & & 2.0390 & 1.2355 \\
\hline & & 2.0371 & 1.2175 \\
\hline & & 2.0379 & 1.2220 \\
\hline & & 2.0462 & 1.2235 \\
\hline \multirow{15}{*}{ Utah } & & 2.0726 & 1.2051 \\
\hline & & 2.0805 & 1.1947 \\
\hline & & 2.0720 & 1.2004 \\
\hline & & 2.0702 & 1.2000 \\
\hline & & 2.0733 & 1.1990 \\
\hline & & 2.0504 & 1.2099 \\
\hline & & 2.0541 & 1.2069 \\
\hline & & 2.0528 & 1.2138 \\
\hline & & 2.0671 & 1.2185 \\
\hline & & 2.0357 & 1.2261 \\
\hline & & 2.0480 & 1.2248 \\
\hline & & 2.0407 & 1.2266 \\
\hline & & 2.0427 & 1.2267 \\
\hline & & 2.0561 & 1.2150 \\
\hline & & 2.0628 & 1.2097 \\
\hline Mean & & 2.0458 & 1.2194 \\
\hline SD & & 0.0158 & 0.0158 \\
\hline \multicolumn{4}{|c|}{ Pristine lake sediments $^{16,17}$} \\
\hline \multirow{3}{*}{ Great Lakes } & & 2.0339 & 1.2240 \\
\hline & & 2.0323 & 1.2241 \\
\hline & & 2.0360 & 1.2227 \\
\hline
\end{tabular}




\begin{tabular}{|c|c|c|c|}
\hline & ${ }^{206} \mathrm{~Pb} /{ }^{204} \mathrm{~Pb}$ & ${ }^{208} \mathrm{~Pb} /{ }^{206} \mathrm{~Pb}$ & ${ }^{206} \mathrm{~Pb} /{ }^{207} \mathrm{~Pb}$ \\
\hline & & 2.0349 & 1.2240 \\
\hline & & 2.0350 & 1.2229 \\
\hline & & 2.0344 & 1.2240 \\
\hline & & 2.0328 & 1.2245 \\
\hline & & 2.0456 & 1.2148 \\
\hline & & 2.0477 & 1.2140 \\
\hline & & 1.9899 & 1.2529 \\
\hline & & 1.9899 & 1.2519 \\
\hline & & 1.9965 & 1.2489 \\
\hline & & 2.0527 & 1.2258 \\
\hline \multirow{12}{*}{ Chesapeake Bay } & & 2.0563 & 1.2058 \\
\hline & & 2.0593 & 1.204 \\
\hline & & 2.0572 & 1.2071 \\
\hline & & 2.0547 & 1.205 \\
\hline & & 2.0521 & 1.2117 \\
\hline & & 2.0534 & 1.2106 \\
\hline & & 2.0547 & 1.2111 \\
\hline & & 2.0521 & 1.2093 \\
\hline & & 2.0525 & 1.2059 \\
\hline & & 2.0576 & 1.2074 \\
\hline & & 2.0521 & 1.2052 \\
\hline & & 2.0572 & 1.2099 \\
\hline Mean & & 2.0408 & 1.2187 \\
\hline SD & & 0.0206 & 0.0144 \\
\hline
\end{tabular}


Table $\mathrm{S} 5: \mathrm{Pb}$ stable isotope ratios $\left({ }^{208} \mathrm{~Pb} /{ }^{206} \mathrm{~Pb},{ }^{206} \mathrm{~Pb} /{ }^{207} \mathrm{~Pb}\right)$, modeled $\mathrm{Pb}$ contributions from the APP fly ash (\%), and percentages of fly ash particles observed in the Sutton sediments and Waccamaw sediments from ref 18.

\begin{tabular}{|c|c|c|c|c|}
\hline Sediment & ${ }^{{ }^{208} \mathbf{P b} / \mathbf{2 0 6}_{\mathbf{P b}}}$ & ${ }^{{ }^{066} \mathbf{P b} / \mathbf{2 0 7} \mathbf{P b}}$ & $\begin{array}{c}\text { Pb from APP fly } \\
\text { ash (\%) }\end{array}$ & $\begin{array}{c}\text { Ash particles in } \\
\text { sediments (\%) }^{\mathbf{1 8}}\end{array}$ \\
\hline Sutton 1 & 2.0455 & 1.2047 & 10.86 & 5 \\
\hline Sutton 3 & 2.0437 & 1.2109 & 54.59 & 89 \\
\hline Sutton 4 & 2.0521 & 1.2075 & 30.59 & 21 \\
\hline Sutton 5 & 2.0416 & 1.2085 & 37.88 & 38.7 \\
\hline Sutton 7 & 2.0477 & 1.2075 & 30.59 & 27.7 \\
\hline Sutton 2 2015 & 2.0432 & 1.2100 & 48.32 & 58.6 \\
\hline Sutton 3 2015 & 2.0469 & 1.2042 & 6.72 & 36.6 \\
\hline Mean & 2.0458 & 1.2076 & 31.37 & \\
\hline Waccamaw 2 & 2.0526 & 1.2030 & & \\
\hline Waccamaw 3 & 2.0529 & 1.2034 & & \\
\hline Mean & 2.0528 & 1.2032 & & \\
\hline
\end{tabular}

*The calculation of $\mathrm{Pb}$ contributions to the Sutton Lake sediments from the APP fly ash was based on the binary mixing model below: ${ }^{19-22}$

$\mathrm{Pb}_{\text {sam }}=100 \% \times\left\{\left[\left({ }^{206} \mathrm{~Pb} /{ }^{207} \mathrm{~Pb}\right)_{\mathrm{sam}}-\left({ }^{206} \mathrm{~Pb} /{ }^{207} \mathrm{~Pb}\right)_{\mathrm{ref}}\right] /\left[\left({ }^{206} \mathrm{~Pb} / 207 \mathrm{~Pb}\right)_{\mathrm{anth}}-\left({ }^{206} \mathrm{~Pb} / 207 \mathrm{~Pb}\right)_{\mathrm{ref}}\right]\right\}$

where $\mathrm{Pb}_{\text {sam }}$ is the contribution of anthropogenic $\mathrm{Pb}$ source in the sample; $\left({ }^{206} \mathrm{~Pb} / 207 \mathrm{~Pb}\right)_{\text {sam }}$ is the measured ratio of the sample; $\left({ }^{206} \mathrm{~Pb} /{ }^{207} \mathrm{~Pb}\right)$ ref is the known ratio of the reference background; $\left({ }^{206} \mathrm{~Pb} /{ }^{207} \mathrm{~Pb}\right)_{\text {anth }}$ is the known ratio of the anthropogenic $\mathrm{Pb}$ source. In this study, $\left({ }^{206} \mathrm{~Pb} /{ }^{207} \mathrm{~Pb}\right)$ sam is the measured ${ }^{206} \mathrm{~Pb} /{ }^{207} \mathrm{~Pb}$ value of each Sutton Lake sediment; $\left({ }^{206} \mathrm{~Pb} /{ }^{207} \mathrm{~Pb}\right)$ anth is represented by the mean ${ }^{206} \mathrm{~Pb} /{ }^{207} \mathrm{~Pb}$ value of APP fly ash (Table S3); $\left({ }^{206} \mathrm{~Pb} /{ }^{207} \mathrm{~Pb}\right)_{\text {ref }}$ is represented by the mean ${ }^{206} \mathrm{~Pb} /{ }^{207} \mathrm{~Pb}$ value of Lake Waccamaw sediments (Table S5). 

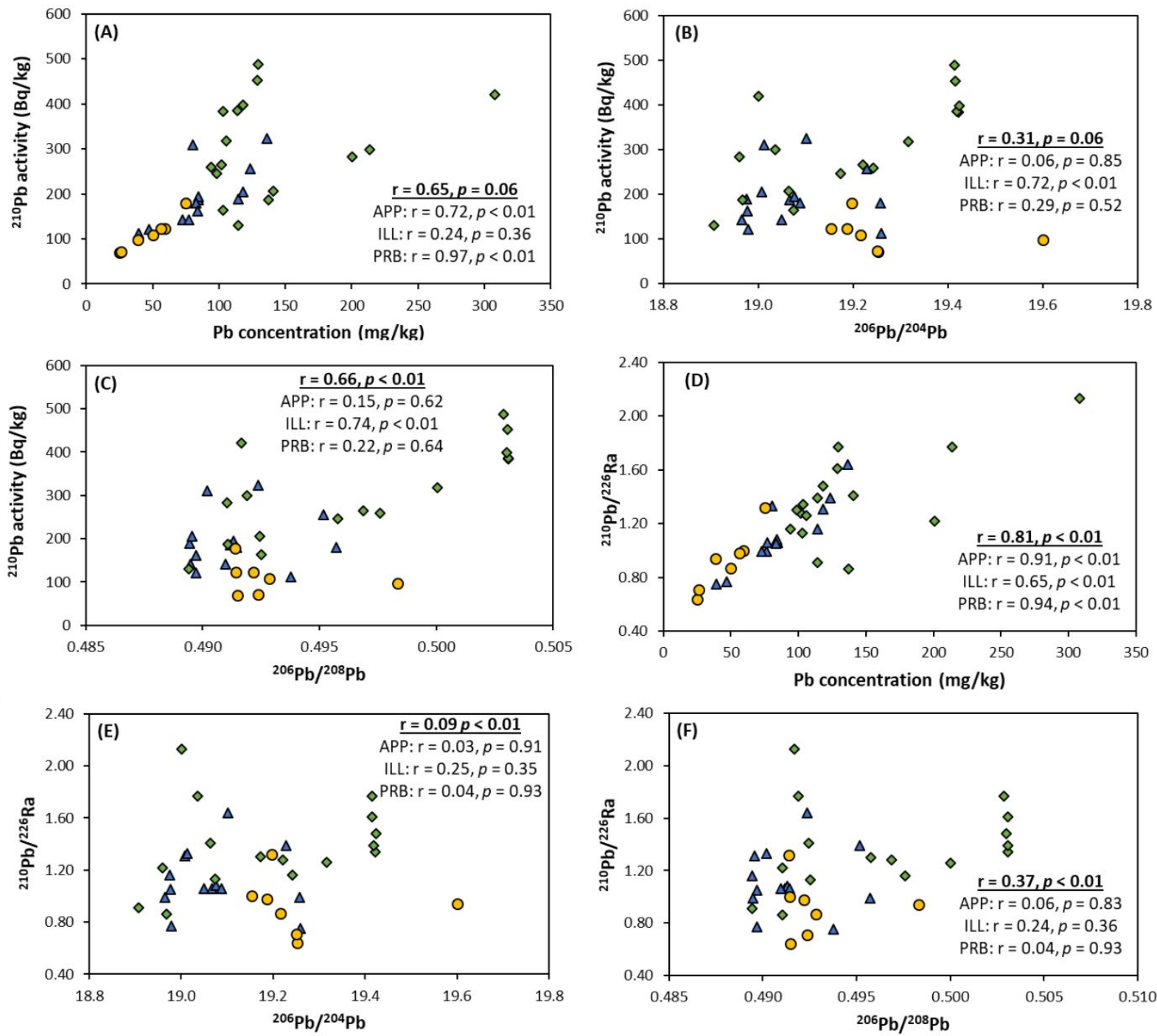

Figure $\mathrm{S} 1$ : Comparisons of $\mathrm{Pb}$ concentrations and $\mathrm{Pb}$ isotope ratios with ${ }^{210} \mathrm{~Pb}$ activities and ${ }^{210} \mathrm{~Pb} /{ }^{226} \mathrm{Ra}$ ratios for fly ash samples in this study (Appalachian, blue triangles; Illinois, green diamonds; Powder River Basin, yellow circles). Regression analysis was repeated both by individual basin and by taking all three basins as a whole. The overall analysis statistics are in bold and underlined. r: Pearson coefficients; p: p-value (at 95\% confidence level). Displayed data are from Table S3. 

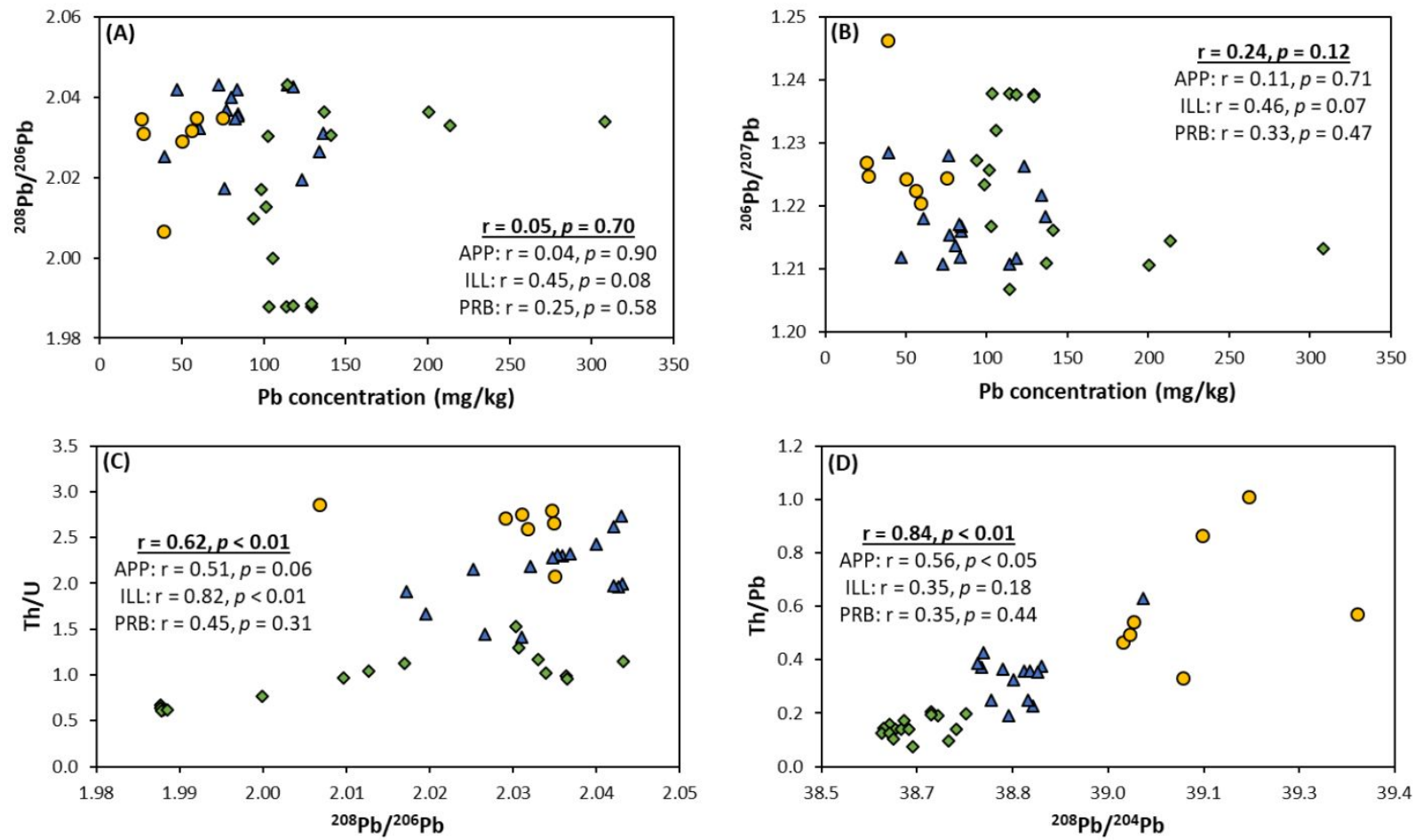

Figure $\mathrm{S} 2$ : Comparisons of $\mathrm{Pb}$ concentration, $\mathrm{Th} / \mathrm{U}$ ratio, and $\mathrm{Th} / \mathrm{Pb}$ ratio with $\mathrm{Pb}$ isotope ratios for fly ash samples in this study (Appalachian, blue triangles; Illinois, green diamonds; Powder River Basin, yellow circles). Regression analyses were repeated both by individual basins and by taking all three basins as a whole. The overall analysis statistics are in bold and underlined. r: Pearson coefficients; p: p-value (at 95\% confidence level). Displayed data are from Table S3. 


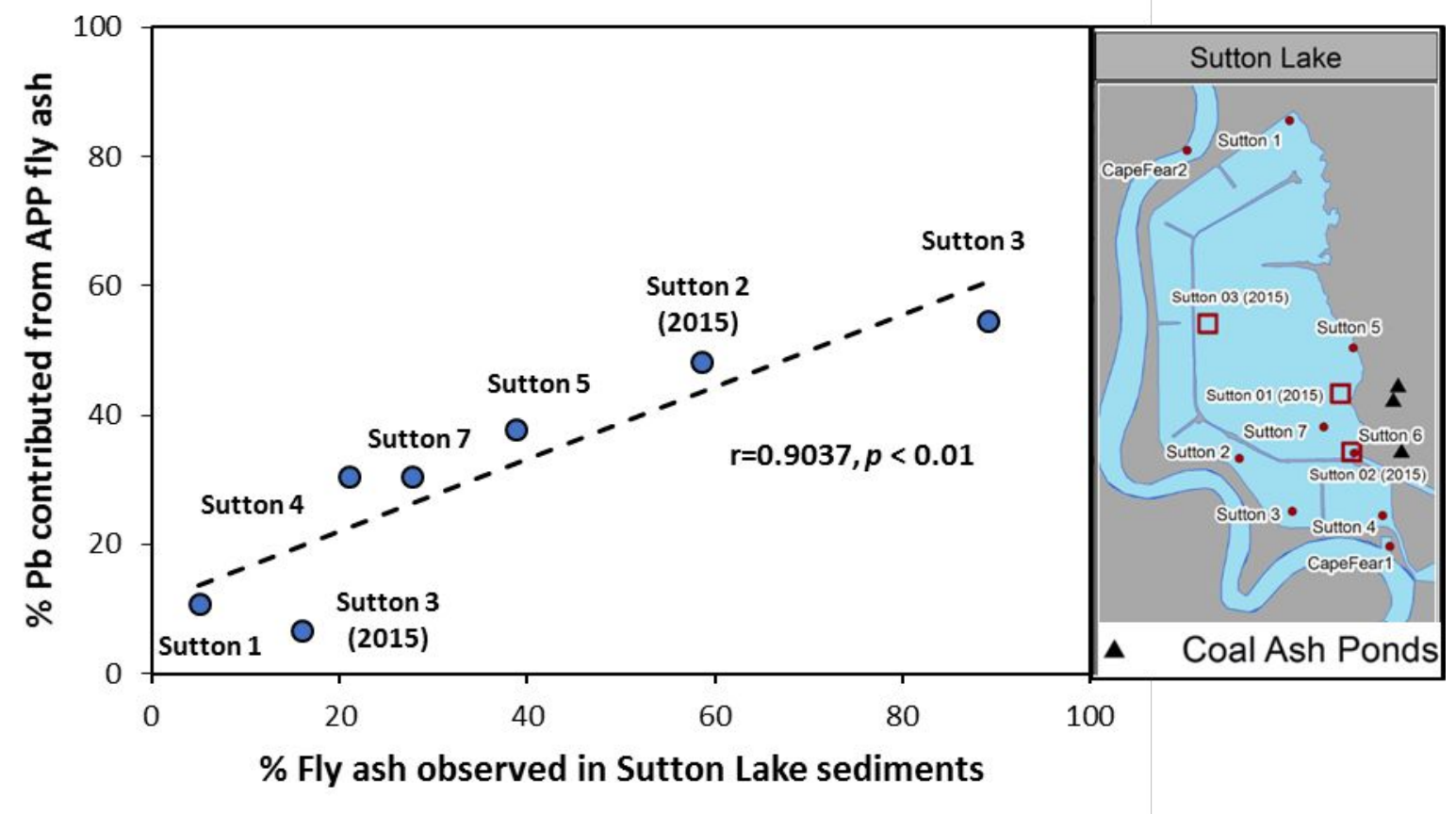

Figure S3: Estimated Pb contributions (\%) from the Appalachian (APP) fly ash to the Sutton Lake sediments versus the percentages of fly ash particles observed in the sediments. $r$ : Pearson coefficients; $p$ : p-value (at 95\% confidence level). The insert lake map was modified from ref 18 and was made with ArcMap (ESRI, ArcMap: Version 10.3.1. Redlands, CA: Environmental Systems Research Institute). 


\section{References:}

(1) Yuan, H.; Yuan, W.; Cheng, C.; Liang, P.; Liu, X.; Dai, M.; Bao, Z.; Zong, C.; Chen, K.; Lai, S. Evaluation of Lead Isotope Compositions of NIST NBS 981 Measured by Thermal Ionization Mass Spectrometer and Multiple-Collector Inductively Coupled Plasma Mass Spectrometer. Solid Earth Sci. 2016, 1 (2), 74-78. DOI: 10.1016/j.sesci.2016.04.001.

(2) Lauer, N. E.; Hower, J. C.; Hsu-Kim, H.; Taggart, R. K.; Vengosh, A. Naturally Occurring Radioactive Materials in Coals and Coal Combustion Residuals in the United States. Environ. Sci. Technol. 2015, 49 (18), 11227-11233. DOI: 10.1021/acs.est.5b01978.

(3) Sangster, D. F.; Outridge, P. M.; Davis, W. J. Stable Lead Isotope Characteristics of Lead Ore Deposits of Environmental Significance. Environ. Rev. 2000, 8 (2), 115-147. DOI: 10.1139/a00008.

(4) Leach, D. L.; Bradley, D. C.; Huston, D.; Pisarevsky, S. A.; Taylor, R. D.; Gardoll, S. J. SedimentHosted Lead-Zinc Deposits in Earth History. Econ. Geol. 2010, 105 (3), 593-625. DOI: 10.2113/gsecongeo.105.3.593.

(5) Chow, T. J.; Johnstone, M. S. Lead Isotopes in Gasoline and Aerosols of Los Angeles Basin, California. Science 1965, 147 (3657), 502-503. DOI: 10.1126/science.147.3657.502.

(6) Sherrell, R. M.; Boyle, E. A.; Hamelin, B. Isotopic Equilibration between Dissolved and Suspended Particulate Lead in the Atlantic Ocean: Evidence from 210Pb and Stable Pb Isotopes. J. Geophys. Res. Oceans 1992, 97 (C7), 11257-11268. DOI: 10.1029/92JC00759.

(7) Dunlap, C. E.; Bouse, R.; Flegal, A. R. Past Leaded Gasoline Emissions as a Nonpoint Source Tracer in Riparian Systems: A Study of River Inputs to San Francisco Bay. Environ. Sci. Technol. 2000, 34 (7), 1211-1215. DOI: 10.1021/es9905905.

(8) Rabinowitz, M. B. Stable Isotope Mass Spectrometry in Childhood Lead Poisoning. Biol. Trace Elem. Res. 1987, 12 (1), 223-229. DOI: 10.1007/BF02796682.

(9) Viczian, M.; Lasztity, A.; Barnes, R. M. Poisoning by Inductively Coupled Plasma Mass Spectrometry. Verification and Case Studies. J. Anal. At. Spectrom. 1990, 5, 293-300. DOI: 10.1039/JA9900500293.

(10) Jaeger, R. J.; Weiss, A. L.; Manton, W. I. Isotopic Ratio Analysis in Residential Lead-Based Paint and Associated Surficial Dust. J. Toxicol. Clin. Toxicol. 1998, 36 (7), 691-703. DOI: $10.3109 / 15563659809162617$.

(11) Bollhöfer, A.; Rosman, K. J. R. Isotopic Source Signatures for Atmospheric Lead: The Northern Hemisphere. Geochim. Cosmochim. Acta 2001, 65 (11), 1727-1740. DOI: 10.1016/S00167037(00)00630-X.

(12) Bollhöfer, A.; Rosman, K. J. R. The Temporal Stability in Lead Isotopic Signatures at Selected Sites in the Southern and Northern Hemispheres. Geochim. Cosmochim. Acta 2002, 66 (8), 1375-1386. DOI: $10.1016 / \mathrm{S} 0016-7037(01) 00862-6$.

(13) Chow, T. J.; Earl, J. L. Lead Isotopes in North American Coals. Science 1972, 176 (4034), 510-511. DOI: $10.1126 /$ science.176.4034.510.

(14) Díaz-Somoano, M.; Kylander, M. E.; López-Antón, M. A.; Suárez-Ruiz, I.; Martínez-Tarazona, M. R.; Ferrat, M.; Kober, B.; Weiss, D. J. Stable Lead Isotope Compositions In Selected Coals From Around The World And Implications For Present Day Aerosol Source Tracing. Environ. Sci. Technol. 2009, 43 (4), 1078-1085. DOI: 10.1021/es801818r.

(15) Reimann, C.; Smith, D. B.; Woodruff, L. G.; Flem, B. Pb-Concentrations and Pb-Isotope Ratios in Soils Collected along an East-West Transect across the United States. Appl. Geochem. 2011, 26 (9), 1623-1631. DOI: 10.1016/j.apgeochem.2011.04.018.

(16) Graney, J. R.; Halliday, A. N.; Keeler, G. J.; Nriagu, J. O.; Robbins, J. A.; Norton, S. A. Isotopic Record of Lead Pollution in Lake Sediments from the Northeastern United States. Geochim. Cosmochim. Acta 1995, 59 (9), 1715-1728. DOI: 10.1016/0016-7037(95)00077-D. 
(17) Marcantonio, F.; Zimmerman, A.; Xu, Y.; Canuel, E. A Pb Isotope Record of Mid-Atlantic US Atmospheric Pb Emissions in Chesapeake Bay Sediments. Mar. Chem. 2002, 77 (2), 123-132. DOI: 10.1016/S0304-4203(01)00081-0.

(18) Vengosh, A.; Cowan, E. A.; Coyte, R. M.; Kondash, A. J.; Wang, Z.; Brandt, J. E.; Dwyer, G. S. Evidence for Unmonitored Coal Ash Spills in Sutton Lake, North Carolina: Implications for Contamination of Lake Ecosystems. Sci. Total Environ. 2019, 686, 1090-1103. DOI: 10.1016/j.scitotenv.2019.05.188.

(19) Monna, F.; Lancelot, J.; Croudace, I. W.; Cundy, A. B.; Lewis, J. T. Pb Isotopic Composition of Airborne Particulate Material from France and the Southern United Kingdom: Implications for $\mathrm{Pb}$ Pollution Sources in Urban Areas. Environ. Sci. Technol. 1997, 31 (8), 2277-2286. DOI: 10.1021/es960870+.

(20) Emmanuel, S.; Erel, Y. Implications from Concentrations and Isotopic Data for Pb Partitioning Processes in Soils. Geochim. Cosmochim. Acta 2002, 66 (14), 2517-2527. DOI: 10.1016/S00167037(02)00863-3.

(21) Ettler, V.; Mihaljevič, M.; Komárek, M. ICP-MS Measurements of Lead Isotopic Ratios in Soils Heavily Contaminated by Lead Smelting: Tracing the Sources of Pollution. Anal. Bioanal. Chem. 2004, 378 (2), 311-317. DOI: 10.1007/s00216-003-2229-y.

(22) Hou, X.; Parent, M.; Savard, M. M.; Tassé, N.; Bégin, C.; Marion, J. Lead Concentrations and Isotope Ratios in the Exchangeable Fraction: Tracing Soil Contamination near a Copper Smelter. Geochem. Explor. Environ. Anal. 2006, 6 (2-3), 229-236. DOI: 10.1144/1467-7873/05-092. 